\title{
Seismic tests of accretion in central stars of planetary systems
}

\author{
Michaël Bazot, Sylvie Vauclair \\ Laboratoire d'Astrophysique, Observatoire Midi-Pyrénées, 14 avenue \\ Edouard Belin, 31400 Toulouse, France
}

\begin{abstract}
Central stars of extra-solar planetary systems are metal-rich. Planet accretion or initial high metallicity are two models proposed to explain this observational fact that can be tested with asteroseismology. We calculate two stellar models, one with accretion and one with high initial metallicity, in order to obtain the same external parameters for both of them. We then compare their internal structures and their oscillation frequencies.
\end{abstract}

\section{Introduction}

Central stars of planetary systems are known to be overabundant in metals (see, e.g., Santos et al. 2001; Santos et al. 2003). Two scenarios have been suggested to explain such an excess. In the first, the protostellar gas is metal-rich and so is the star. The high density of heavy elements, compared to stars without planets, increases the probability of planetesimal formation, and thus of planet formation. The second explanation assumes that planets are formed out of gas with solarlike metallicity. The central stars accrete hydrogen poor-matter during, and after planetary formation, thereby enhancing their metallicities in their outer layers (Murray et al. 2001). We suggest that these two scenarios can be tested by asteroseismology. Here we analyse and compare the oscillation frequencies in stellar models with the same external parameters, but with different histories: with and without accretion.

\section{Calibration}

We computed 1.1- $\mathrm{M}_{\odot}$ stellar models with the Toulouse-Geneva code of stellar evolution. One of them has a high initial metallicity, from the surface to the core, of $Z=0.0443$. The other has initial solar abundances, but it experienced accretion at the beginning of its main-sequence evolution. Both models include microscopic diffusion. We adjusted the mass of accreted material $\left(\mathrm{M}_{a}\right)$, the mixing-length parameter $(\alpha)$ and the initial helium abundance $\left(\mathrm{Y}_{0}\right)$, which are input parameters in our stellar evolution code, in order to obtain stars with the same effective temperature $\left(\mathrm{T}_{\text {eff }}\right)$, luminosity $(\mathrm{L})$ and metallicity $(\mathrm{Z})$. Input and output parameters for these models are given in Tables 1 and 2; evolutionary tracks are represented in Fig. 1. 


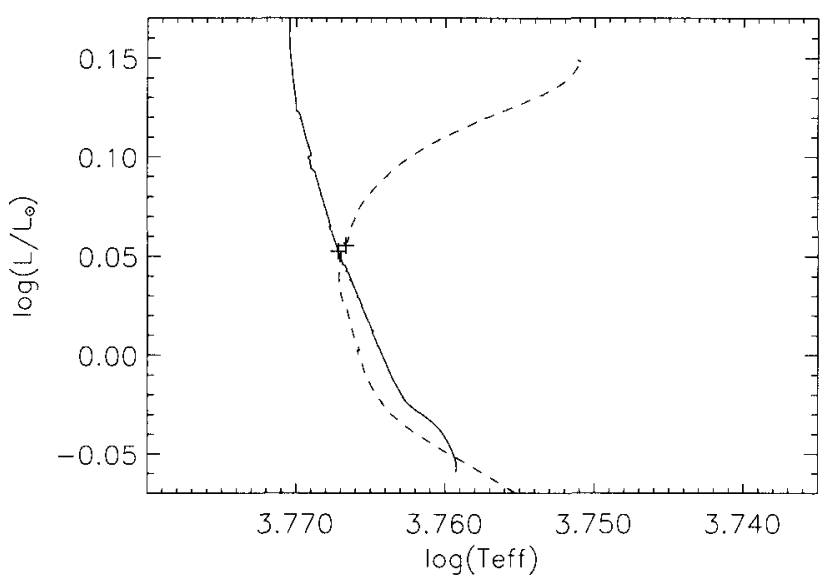

Figure 1. Evolutionary tracks for a star experiencing accretion of planetary material (full line) and a supermetallic star (dashed line). The models used for this study are marked with a cross on each track.

The accreted mass is composed of elements heavier than helium with the same relative abundances as in the Sun. It is given in units of $\mathrm{M}_{J u p}(=1.9 \times$ $10^{27} \mathrm{~kg}$ ). When the convergence of two models is obtained, we compute their oscillation frequencies. We used the adiabatic pulsation code described by Brassard et al. (1992).

Table 1. Input parameters for model 1 (with accretion) and model 2 (supermetallic). The mass fractions $\mathrm{X}_{0}, \mathrm{Y}_{0}, \mathrm{Z}_{0}$ are the surface quantities; $\alpha$ is the mixing-length parameter.

\begin{tabular}{lcccccc}
\hline Model & $\mathrm{M}_{\star}\left(\mathrm{M}_{\odot}\right)$ & $\mathrm{M}_{a}\left(\mathrm{M}_{\text {Jup }}\right)$ & $\alpha$ & $\mathrm{X}_{0}$ & $\mathrm{Y}_{0}$ & $\mathrm{Z}_{0}$ \\
\hline 1 & 1.1 & 0.5 & 2.08 & 0.721 & 0.260 & 0.019 \\
2 & 1.1 & - & 2.08 & 0.626 & 0.330 & 0.044 \\
\hline \hline
\end{tabular}

\section{Results}

\subsection{Physical parameters of the stars}

As the physical processes in the stars are not the same, the internal structures of models with the same external parameters present detectable differences. In Fig. 2 we plot the relative differences of temperature, pressure, density and the square of the sound speed for the point on the HR diagram (see Fig. 1) at which the stars have similar external parameters. In Table $2 R_{c v}$ is the radius at the bottom of the outer convective zone, $R_{\star}$ is the star's radius. 
Table 2. Output parameters for model 1 (with accretion) and model 2 (supermetallic). The mass fractions $\mathrm{X}, \mathrm{Y}, \mathrm{Z}$ are the surface quantities. Model 1 has a convective core; model 2 does not.

\begin{tabular}{ccccccccc}
\hline Model & $\begin{array}{c}\text { Age } \\
\text { Gyr }\end{array}$ & $\mathrm{X}$ & $\mathrm{Y}$ & $\mathrm{Z}$ & $\begin{array}{c}\mathrm{T}_{\text {eff }} \\
\mathrm{K}\end{array}$ & $\frac{L}{L_{\odot}}$ & $\frac{R}{R_{\odot}}$ & $\frac{R_{c v}}{R_{*}}$ \\
\hline 1 & 2.12 & 0.721 & 0.239 & 0.048 & 5850 & 1.128 & 1.037 & 0.709 \\
2 & 2.97 & 0.654 & 0.306 & 0.040 & 5847 & 1.136 & 1.042 & 0.704 \\
\hline \hline
\end{tabular}
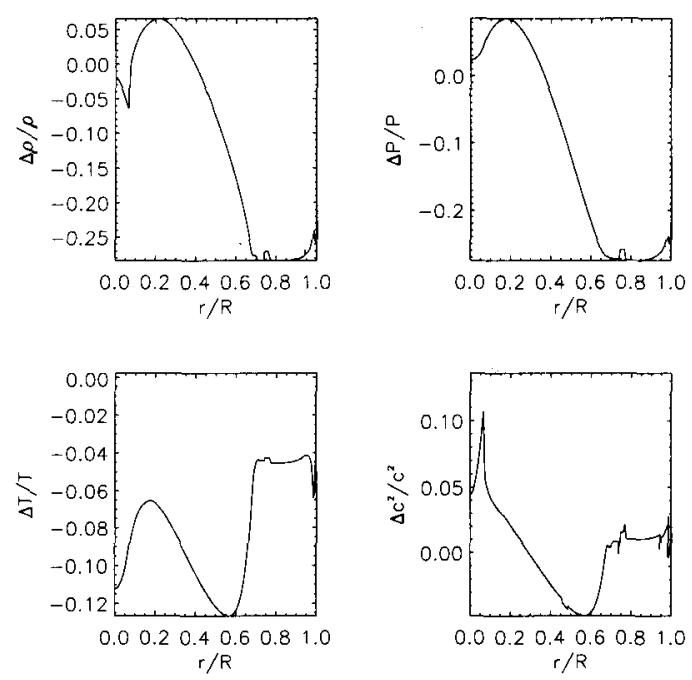

Figure 2. Relative differences in density, pressure, temperature and sound speed. For example $\left.\frac{\Delta T}{T}=\left(T_{1}-T_{2}\right) / T_{2}\right)$, where 1 refers to the accretion model, and 2 refers to the supermetallic model.

\subsection{Oscillation frequencies}

We computed the oscillation frequencies of our models for different values of the azimuthal degree $\ell$ (from 0 to 3 ) and radial order $n$. We then compared the oscillation frequencies. The relative difference between oscillation frequencies, as functions of the radial order and the frequency itself, is shown in Fig. 3.

\section{Discussion}

Sound speed differences, which appear in Fig. 2, are worth studying. They reach $0.5 \%$ below the convective zone and $1 \%$ near the centre. Below the convective zone they reflect differences in temperature, chemical abundances and variation in the mean molecular weight, $\mu$. The peak at $\sim 0.05 R_{\star}$ is due to the presence of a convective core in the supermetallic star. Fig. 3 shows the differences of 


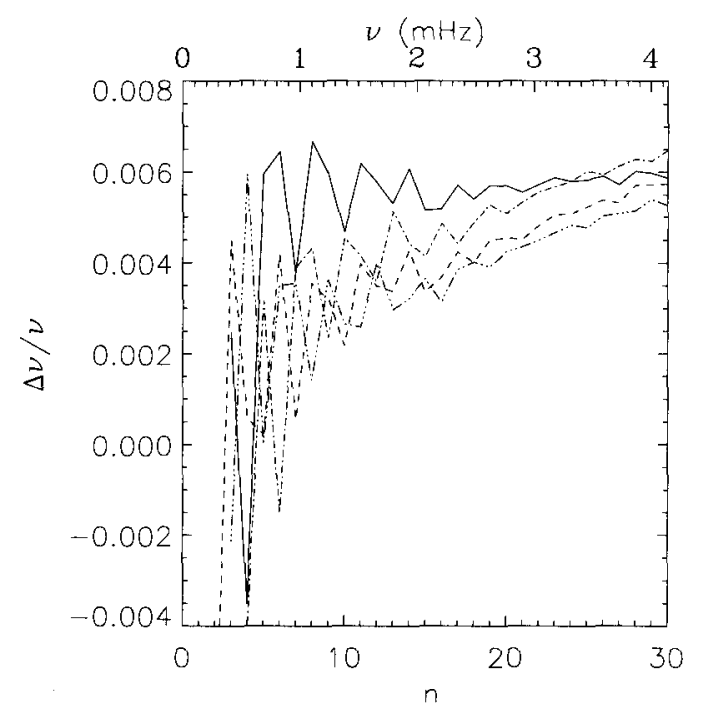

Figure 3. Relative differences of the oscillation frequencies for azimuthal degrees $\ell=0$ (full line), $\ell=1$ (dot-dashed line), $\ell=2$ (dashed line) and $\ell=3$ (double dotted-dashed line).

adiabatic oscillation frequencies linked to the differences in sound speed. They are of the order of a few tenths of percent which is an observable effect with the means of asteroseismology (Bouchy \& Carrier 2002).

A further level in analysis consists in computing the small and second differences for both stars, i.e. $\delta \nu_{n, \ell}=\nu_{n, \ell}-\nu_{n-1, \ell+2}$ and $\delta_{2} \nu=\nu_{n+1}-2 \nu_{n}+\nu_{n-1}$. The former is a good test for stellar interior near the core (Ulrich 1986); the latter is a good indicator for the region near the convective zone (Gough 1990). Computations of these quantities are in process and will be discussed in a forthcoming paper.

\section{References}

Bouchy, F., Carrier, F. 2002, A\&A, 390, 205

Brassard, P., Pelletier, C., Fontaine, G., Wesemael, F. 1992, ApJS, 80, 725

Gough, D.O. 1990, Lect. Notes Phys., Vol. 367: Progress of Seismology of the Sun and Stars, 283

Murray, N., Chaboyer, B., Arras, P., Hansen, B., Noyes, R.W. 2001, ApJ, 555, 801

Santos, N.C., Israelian, G., Mayor, M. 2001, A\&A, 373, 1019

Santos, N.C., Israelian, G., Mayor, M., Rebolo, R., Udry, S. 2003, A\&A, 398, 363

Ulrich, R.K. 1986, ApJ, 306, L37 